\title{
Weitere Therapiefortschritte in Sicht
}

Mit dem BRAF-Inhibitor Vemurafenib ${ }^{1}$ wurde ein Durchbruch beim metastasierten malignen Melanom erzielt. Nun gibt es Hoffnungen, durch die zusätzliche Behandlung mit einem Mitogen-Activated Extracellular Signal Regulated Kinase (MEK)-Inhibitor noch deutlich bessere Therapieergebnisse erzielen zu können.

Welche enormen Fortschritte beim metastasierten Melanom bereits gemacht wurden, hat Dr. Peter Mohr, Buxtehude, bei einem Seminar ${ }^{2}$ dargelegt. So lag das mediane Überleben laut einer Studie aus 2008 bei 6,2 Monaten. Nur 25,5\% der Patienten lebten ein Jahr nach der Diagnose noch, berichtete der Mediziner.

Einen Durchbruch bei der Therapie hat nach seinen Worten Vemurafenib als erster Vertreter des Konzeptes der „Targeted Therapy“ beim malignen Melanom gebracht. Der Wirkstoff ist zur Behandlung des BRAF-V600-Mutations-positiven, nicht resezierbaren oder metasta- sierten Melanoms zugelassen. Bei rund jedem 2. Melanom-Patienten liegt nach Mohr eine solche BRAF-Mutation vor, was eine unkontrollierte Aktivierung des Mitogen-Activated Protein Kinase (MAPK)Signalwegs und damit ein unkontrolliertes Tumorwachstum zur Folge hat. Werden die Patienten mit Vemurafenib behandelt, so führt das Studien zufolge zu einer deutlichen Verlängerung des Gesamtüberlebens von median 9,7 Monaten unter Dacarbazin auf 13,6 Monate unter Vemurafenib $^{3}$. Den Studiendaten zufolge leben nach einem Jahr dadurch noch 58\% und auch nach 2 Jahren noch $32 \%$ der Patienten, berichtete Mohr. Er hob dort ferner das beeindruckend rasche Ansprechen der Patienten hervor: „Wir sehen Fälle, in denen zuvor opioidpflichtige Patienten nach wenigen Wochen keinerlei Schmerzmedikation mehr benötigen.“

Das metastasierte maligne Melanom ist jedoch nach wie vor ein schwer therapierbarer Tumor, sodass weitere Therapiefort- schritte dringend notwendig sind. Die Hoffnungen richten sich zurzeit v.a. auf die Kombination des BRAF-Inhibitors mit einem Hemmstoff des Zellproteins MEK, das ebenfalls Teil des MAPK-Signalwegs ist. Durch die MEK-Inhibition kann den vorliegenden Befunden zufolge eine BRAF-Inhibitor-Resistenz als Folge der Reaktivierung des MAPK-Signalwegs überwunden werden. Der MEK-Inhibitor kann dabei ergänzend zur direkten Hemmung durch Vemurafenib eine indirekte Hemmung des aktivierten BRAF erwirken. Erste Studienergebnisse ${ }^{4}$ belegen laut Mohr ein verbessertes progressionsfreies sowie ein verbessertes Gesamtüberleben durch die Kombination der beiden Wirkprinzipien.

\section{Christine Vetter, Köln}

${ }^{1}$ Zelboraf $^{\circledR}$, Roche Pharma AG, Grenzach-Wyhlen
${ }^{2}$ Mittagsseminar „Der Kampf gegen Hautkrebs:
Neue Meilensteine in der zielgerichteten Thera-
pie“ bei der 48 . DDG-Tagung, Berlin, Mai 2015,
Veranstalter: Roche Pharma AG
${ }^{3}$ Chapman PB et al. J Clin Oncol 2012; 30 (Suppl):
Abstr. 8502
${ }^{4}$ Larkin J et al. N Engl J Med 2014; 371: 1867 - 1876

ziell für Psoriasis-Patienten. Dr. Sascha Gerdes, Kiel, stellte ein online-gestütztes, mit der DGE erarbeitetes Selbstlern-Ernährungsprogramm vor, mit dem das Therapiemanagement der Psoriasis optimiert werden kann. Das Programm soll zur Bewegung und zum gesunden Abnehmen motivieren und hat in ersten, noch nicht veröffentlichten Erhebungen unter Teilnehmern schon messbare Effekte gezeigt, berichtete Gerdes.

Mittlerweile werden Biologika von der aktuellen S3-Leitlinie der DDG bei mittelschwerer und schwerer Psoriasis vulgaris empfohlen.

\section{Elke Klug, Berlin}

\footnotetext{
1 Stelara ${ }^{\circledR}$, Janssen-Cilag GmbH, Neuss

2 Symposium „Psoriasis kommt selten allein Blickpunkt Komorbidität“ bei der 48. DDG-Tagung, Berlin, April 2015. Veranstalter: JanssenCilag $\mathrm{GmbH}$

${ }^{3}$ Hugh J et al. J Am Acad Dermatol 2014; 70 : 168-177

${ }^{4}$ Guida et al. Clin Nutr 2014; 33: 399-405

${ }^{5}$ Naldi L et al. Br J Dermatol 2014; 170: 634-642
}

Gewichtsabnahme hilft zusätzlich Umgekehrt hat die zur gezielten PsoriasisBehandlung ergänzende Korrektur der Risikofaktoren wie Adipositas einen positiven Effekt auf die Psoriasis-Manifestationen ${ }^{4,5}$. Deshalb ist auch die Empfehlung eines Abnehmprogramms ein sinnvoller Bestandteil der Therapiestrategie bei Psoriasis. Gewichtsabnahme, insbesondere die Reduktion des Bauchfettes, ist essen-
Nach Angaben der Industrie 